\title{
A Built-up Virtual Laboratory to Enhance Understanding of Concrete Structure Design Requirement
}

\author{
http://dx.doi.org/10.3991/ijoe.v12i03.5462 \\ S. H. Park ${ }^{1}$, A. D. Adeba ${ }^{1}$, Y. K. Hwang ${ }^{1}$, J. E. Bolander ${ }^{2}$, Y. M. Lim ${ }^{1}$ \\ 1 Yonsei University, Seoul, Korea \\ ${ }^{2}$ University of California Davis, Davis, USA
}

\begin{abstract}
In this paper, simulation tools for educational purposes are developed to enhance understanding in undergraduate students taking courses related to concrete structural behavior. As undergraduate students learn about concrete structure, they commonly reach limits regarding their understanding of failure behavior in materials. Design codes should therefore be followed when students learn about reinforced concrete (RC) design. Students are able to follow a step-by-step design procedure for a $\mathrm{RC}$ beam using simple calculations. However, they usually then face some requirement, such as a design code, that requires students to make an RC beam with several pieces of rebar instead of one piece, because this provides a more desirable behavior for the RC structure. Understanding why the code is required needs more than just reading. By conducting an experiment, students can easily understand the reason for the code. However, real experiments for concrete structures are time-consuming and costly; therefore, provision of a realistic simulation tool, the 'Virtual Concrete Structure Laboratory,' for engineering educational purposes allows students to obtain virtual hands-on experience to learn about concrete structure. The main purpose of this paper is to show the effectiveness of this virtual lab in civil engineering education.
\end{abstract}

Index Terms-concrete structural behavior, hands-on experience, reinforced concrete structure, virtual laboratory

\section{INTRODUCTION}

Experiments and laboratory work are considered important components of engineering education, and many studies have investigated the development of virtual labs for conducting experiments. Two approaches are usually taken when conducting virtual labs: remote and simulation labs. Remote labs allow the students to work on real equipment and instrumentation in the laboratory from a long distance, whereas simulation labs are used to explain and reinforce complicated concepts or phenomena that are not easily visualized. However, both lab types have some limitations; for example, a remote lab should be open 24 hours every day, which can be expensive, whereas simulation does not provides real results. Many attempts are now being made to capitalize on recent advancements in computer and web technology to overcome these issues, by developing alternatives that can replace real laboratory work with computers [1].

One of the main alternatives is the development of simulations that generate almost the same results as a real experiment. Simulations that behave like real experiments would be significant teaching tools for engineering fields where experiments play an important role, such as civil engineering.

In the civil engineering field, concrete is one of the most widely used materials. However, concrete is not an easy material to understand in terms of its failure behavior. Cracks in concrete, which are difficult to predict, can cause behaviors such as sudden failure or even collapses of structures. The difficulty in concrete crack prediction arises because of the heterogeneity of concrete, which is composed of cement, water, and aggregates. Therefore, the behaviors of failure depend on the local characteristics; and propagation of cracks is directly influenced by locations, sizes, and characteristics of aggregates [2].

The tensile strength of concrete is about ten percent of its compressive strength. And concrete has lower fracture energy, showing quasi-brittle failure behavior. Hence, cracks of different degrees and forms occur during laboratory tests [3]. Concrete, therefore, requires reinforcement to overcome its weakness in tension side. Generally, reinforcement entails the use of steel reinforcing bars (rebar), which are placed into the tension side of concrete structures to resist the opening of cracks. Concrete with this type of reinforcement is called reinforced concrete (RC).

The RC design requirement is one of the main subjects of study by civil engineering students. Designing an RC structure is not difficult, so students can readily follow the design procedure. However, understanding why some requirements are needed is another story. Some of the requirements are difficult to understand theoretically because they are based on large number of experimental results. Therefore, students often need direct experience to understand the design requirements. The phrase "A picture is worth a thousand words" is applicable in education methodology.

Experiments are an efficient way to understand subjects that are difficult to grasp using only textbooks. This is the main reason why experimental work is essential in undergraduate courses in engineering education [4]. However, experiments demand a lot of time and money. For example, running a three-point bending test with concrete requires that many things be considered. Preparing and curing a concrete beam takes at least a month. The test equipment to be used on the cured concrete should be handled by an expert, as a great deal of care is needed to perform the experiment [5]. Therefore, providing a good experimental environment for undergraduate students is challenging. 
The difficulties of conducting a real experiment can be overcome by using the 'Virtual Concrete Structure Laboratory' as a solution. The main purpose of this virtual lab is to provide simulation tools that can substitute for real experiments. Crack behavior of concrete was analyzed in the present study using the simulation programs based on the Rigid-Body-Spring-Network (RBSN) model. RBSN is attractive for its simplicity, freedom in mesh layout and generation, and provision of a discrete representation of material disorder and failure. This system is used for analysis of fractures in heterogeneous materials such as concrete [6].

The objective of this paper is to develop a 'Virtual Concrete Structure Laboratory' to enhance student understanding of RC behavior. The paper is presented in the following order: introduction of the numerical model used for analysis, explanation of the process of a virtual lab, examples of simulations, and expectations of the virtual lab's effectiveness based on the responses of the students.

\section{Numerical Model [4]}

\section{A. Modeling Concrete with RBSN}

This study adopted RBSN, one of the random lattice models. In RBSN, concrete is considered as a group of rigid cells called Voronoi cells. Between two Voronoi cells is a spring set and these springs express the overall behavior of concrete [6]. The general mesh generation process is described in Fig. 1: (a) points are randomly generated in given domain, (b) from the generated points, Delaunay triangles are created, (c) after Delaunay triangulation, the mesh can be transferred to the Voronoi diagram [4]. The concrete structures are generated by this process.

\section{B. Modeling Rebar with a Semi-discrete Method}

Rebar is modeled with a semi-discrete method [7], [8], which generates rebars independently in a mesh configuration. The rebar crosses the facets of the Voronoi diagram and contributes to the stiffness of the RBSN elements. This semi-discrete method adds no additional degrees of freedom to the system. Therefore, the simulation can be run with greater efficiency. Fig. 2 shows a reinforcing element within a two-cell assembly of a lattice model [4].

\section{VirTUAL CONCRETE STRUCTURE LABORATORY}

Fig. 3 shows the testing process for the "Virtual Concrete Structure Laboratory." A test is performed by stepby-step application of mesh generation, analysis, and post processing programs. For each step, inputs are needed and a portion of the outputs is used as inputs for the next step. First, the users select the geometry of specimens as inputs for the mesh generation program. When the mesh is generated, a decision is made regarding the boundary conditions and material properties to be used to run the analysis program. After the analysis, the users can obtain the results (deformation, fracture, load-displacement curve, visualized shape, etc.) using the post processing program. This system can be accessed both by researchers and by students. Students can access the website hosting the virtual laboratory platform from anywhere and at any time. The Graphic User Interface (GUI) environment and guidelines are provided on the website [4].

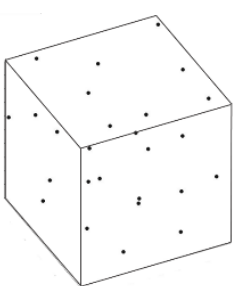

(a) Generated point

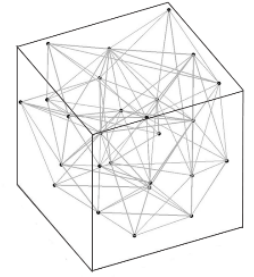

(b) Delaunay triangulation

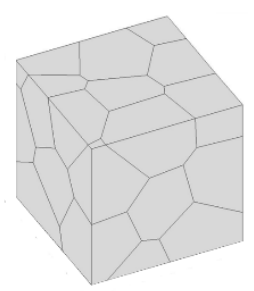

(c) Voronoi diagram
Figure 1. Mesh generation process [7].

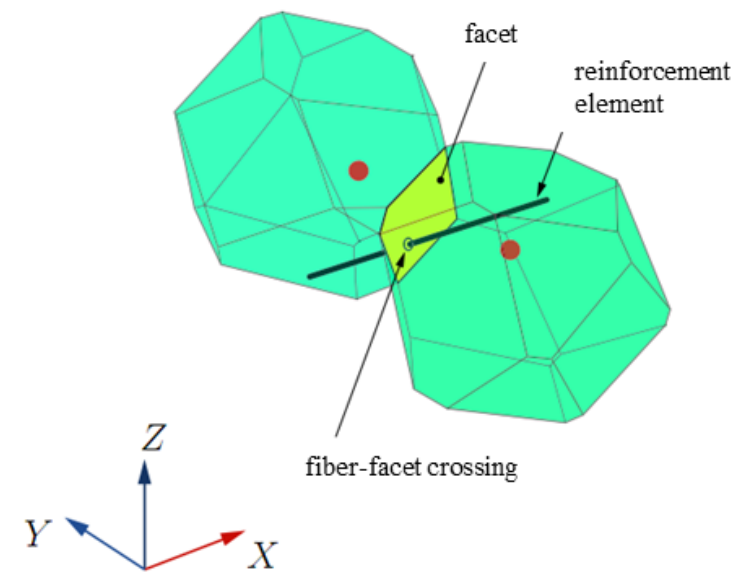

Figure 2. Reinforcing element within a two-cell assembly of a lattice model [7]

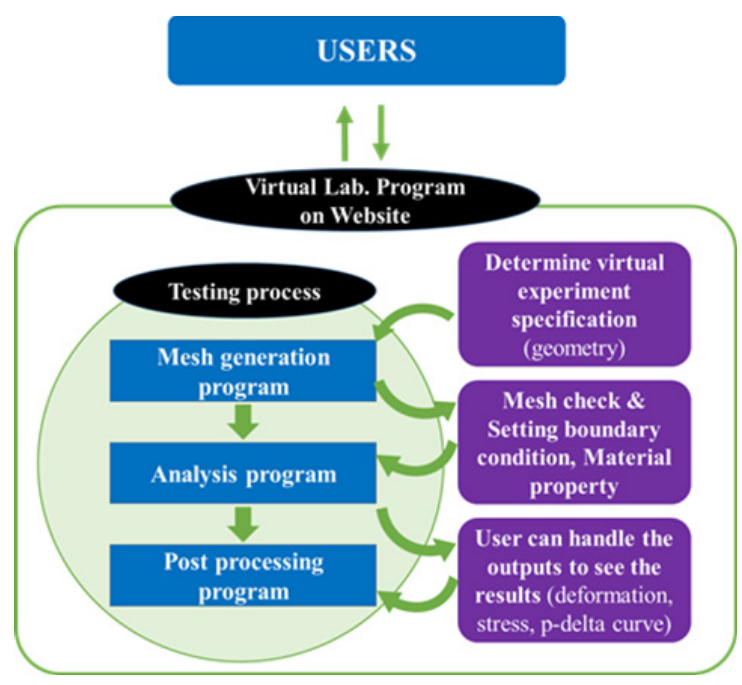

Figure 3. The process of a virtual laboratory experiment [4].

\section{Simulation}

Linear structural analysis, concrete failure analysis, and $\mathrm{RC}$ analysis of rebar distribution effects are introduced according to the general curriculum of civil engineering education. In this study, the geometry and basic information from the previous technical literature were adopted to analyze a three-point bending test of a concrete beam and a four-point bending test of an RC beam $[9,10]$.

\section{A. Linear structural Analysis}

Undergraduate students studying basic subjects, such as the mechanics of materials, learn about the deflection of beams as one of the main subjects taught in class [11]. Students have to use an analytical solution to find the deflection at some point. With that solution, the students can draw an approximate deflection curve. Beam deflec- 
tion can be visualized with a linear structural analysis program, which could help students with the analysis of the beam to see the deflection and obtain a loaddisplacement curve. The students can then compare their results, to check whether they are solving their problem correctly.

A three-point bending test (Fig. 4(a)) was conducted for verification. Fig. 4(c) shows the deformed shape of the simulated beam. Fig. 5 and Fig. 6 are the deflection curve and load-displacement curve, respectively, for the comparison between the linear structural analysis and the analytical solution. Fig. 5 and Fig. 6 show the same results.

\section{B. Concrete Failure Analysis}

The RBSN model was adopted for concrete failure analysis. The mesh of this model is generated according to the Voronoi discretization of the material domain, based on an irregular set of points, and the elemental stiffness in RBSN model is scaled by the geometry of the rigid-bodyspring elements. This irregular lattice model exhibits an artificial heterogeneity, which has strong implications toward its ability to model fractures in concrete [12].

The fracture behavior of concrete is difficult for undergraduate students to understand. Demonstrating realistic phenomena with concrete failure analysis allows students to appreciate the behavior of concrete structures.

The behavior of concrete was checked by simulating a three-point bending test (Fig. 4(a)) for a concrete beam. Fig. 7 shows the failure configurations of the two concrete beams, having same geometry with different meshes. As expected from the tensile strength, main cracks occur at the central bottom part of the beam causing two beams to fail in flexural manner. However, as depicted in enlarged parts of Fig. 7, the detailed crack patterns in two beams are different due to contrasting meshes. The result has similarity with real experiments. Location and direction of crack propagation are similar; however, surfaces of crack in detail are distinct in every specimens with equivalent sizes and properties due to its nonhomogeneous characteristics of concrete. From the fact, numerical model can provide more realistic experiences to students.

\section{RC Analysis of the Rebar Distribution Effect}

Concrete is a quasi-brittle material that undergoes sudden failure. This sudden failure is prevented using rebar to reinforce the concrete. The placement of rebar inside the tensile side of the concrete increases the ductility. When students design an $\mathrm{RC}$ beam, the design code requires the use of distributed rebars instead of one rebar of the same cross-sectional area at the final stage. As the rebar is distributed, the number of cracks increases, but the size of each crack decreases. In other words, the RC becomes more ductile with the distribution of the rebars.

This behavior is demonstrated by simulating three RC beam cases where the RC has the same cross sectional area but different numbers of rebars $(1,3$, or 6$)$. Table I shows the material properties of the concrete and rebar used in this RC analysis. Fig. 8 shows the RC structure and boundary condition for a four-point bending test. The total cross sectional area of the rebar $(A s)$ is about 398 $\mathrm{mm}^{2}$. In case 1 , the diameter of one rebar is $22.52 \mathrm{~mm}$ and the number of rebars is one. In case 2, the diameter of one rebar is $13.00 \mathrm{~mm}$ and the number of rebars is three. In case 3, the diameter of one rebar is $9.19 \mathrm{~mm}$ and the number of rebars is six. Therefore, the total area of reinforce- ment is same in all cases, while number of the rebars used is different. Fig. 9 shows the mesh configuration of RC beam for three cases. Fig. 10 shows the simulation results. The enlarged part of the figure shows the damaged zones with cracks during a loading test. As the number of rebars increases, the damaged zone spreads throughout the tensile side of RC beam, implicating more material are involved in resisting the load. Hence the resistance of the beam increases. As identified in the results, even though the cross sectional area of the rebars is same, behaviors vary depending on the number of rebars. Therefore, the simulation results can teach the students about rebar distribution effects, and why the design code stipulates these requirements [4].

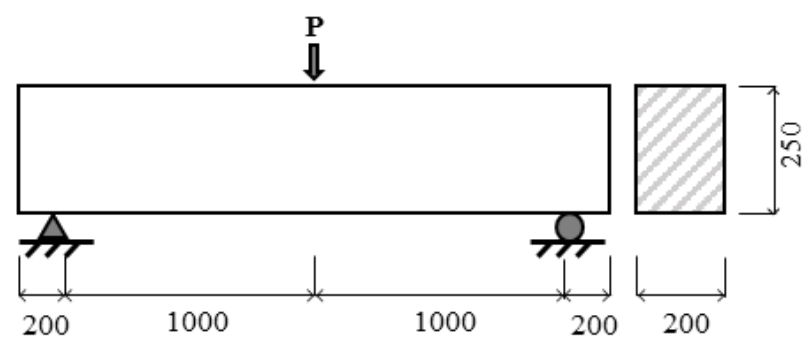

(a) Test specimen configuration (unit : $\mathrm{mm}$ )

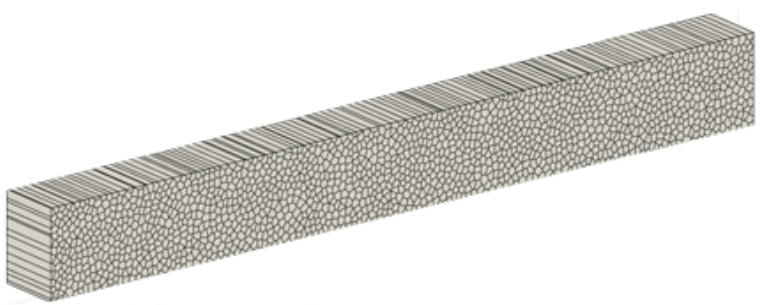

(b) Undeformed shape of simulated beam

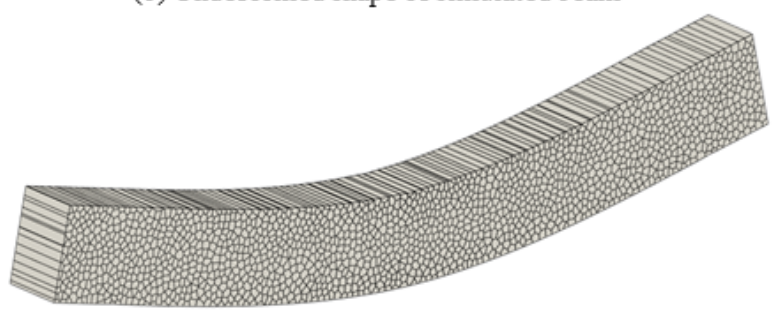

(c) Deformed shape of simulated beam with linear analysis program

Figure 4. Three-point bending test (unit: $\mathrm{mm}$ )

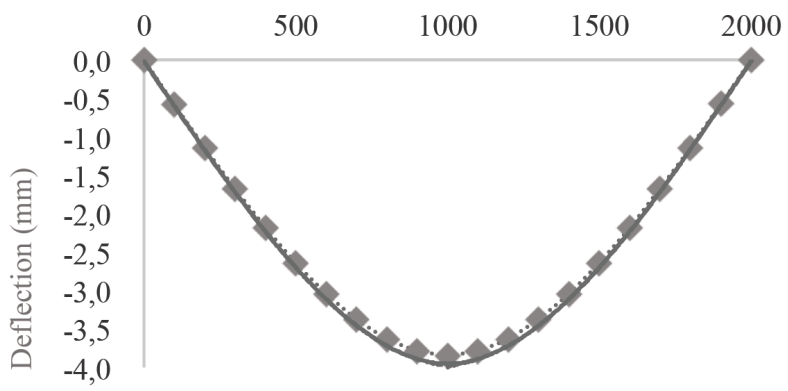

Beam span length (mm)

…...... Analytical solution $\longrightarrow$ RBSN linear analysis

Figure 5. Deflection curve: comparison between the linear structural analysis and the analytical solution. 


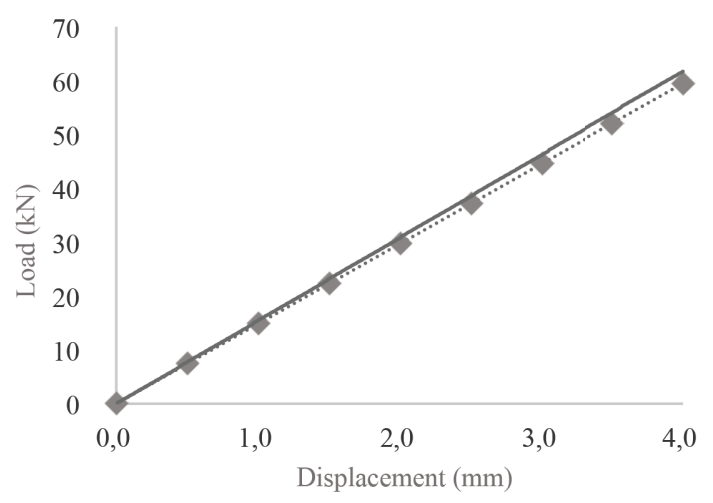

.......... Analytical solution $\longrightarrow$ RBSN linear analysis

Figure 6. Load-displacement curve: comparison between the linear structural analysis and the analytical solution.

(a)

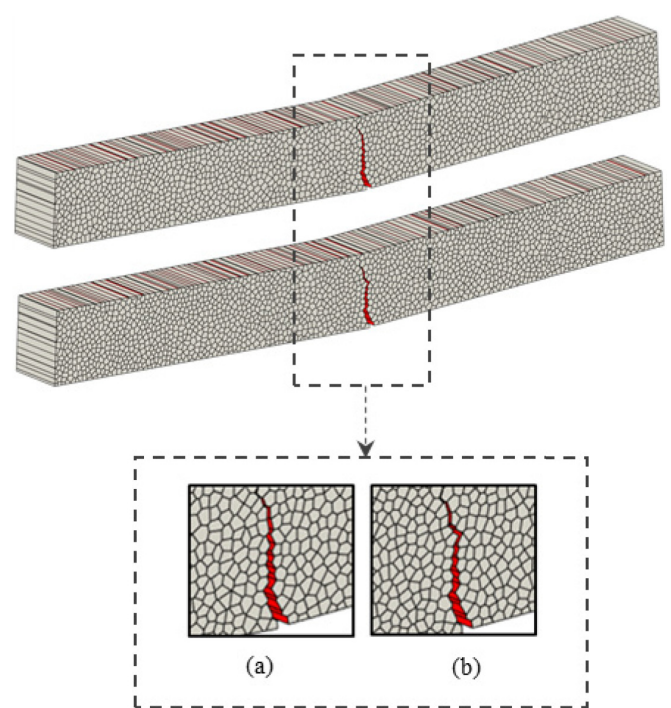

Figure 7. Concrete failure behavior: Two similarly sized beams show two different shapes for the crack

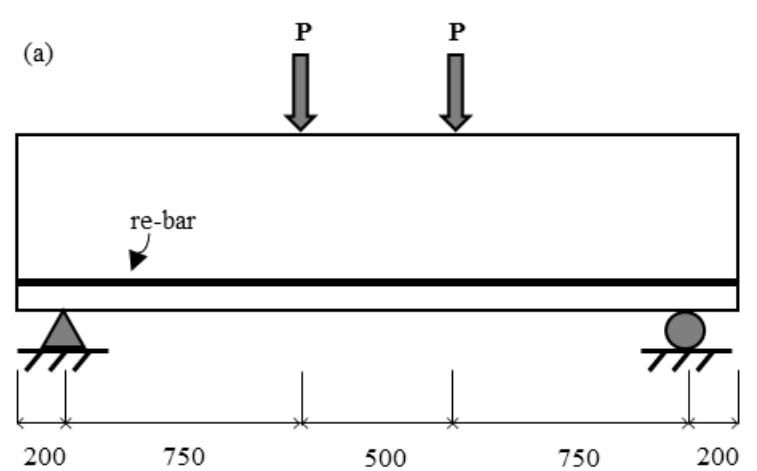

(b)

(c)

(d)

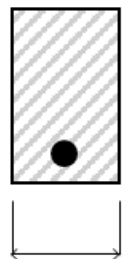

100@2

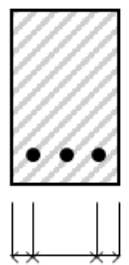

3070@230

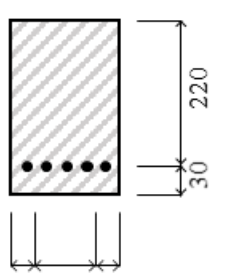

3028@530

Figure 8. RC structure and boundary conditions for four-point-bending test (unit: $\mathrm{mm}$ ).

TABLE I.

PROPERTIES OF MATERIALS [4]

\begin{tabular}{|c|c|c|}
\hline Material & Property & Magnitude \\
\hline \multirow{4}{*}{ Concrete } & Tensile strength, $f_{t}$ & $3.29 \mathrm{MPa}$ \\
\cline { 2 - 3 } & Elasticity, $E_{c}$ & $25.4 \mathrm{GPa}$ \\
\cline { 2 - 3 } & Fracture energy, $G_{f}$ & $81.1 \mathrm{~N} / \mathrm{m}$ \\
\cline { 2 - 3 } & Poisson's ratio, $v$ & 0.19 \\
\hline \multirow{3}{*}{ Rebar } & Elasticity, $E_{s}$ & $186.0 \mathrm{GPa}$ \\
\cline { 2 - 3 } & Yield stress, $\mathrm{f}_{y}$ & $350.0 \mathrm{MPa}$ \\
\hline
\end{tabular}

(a)

(b)

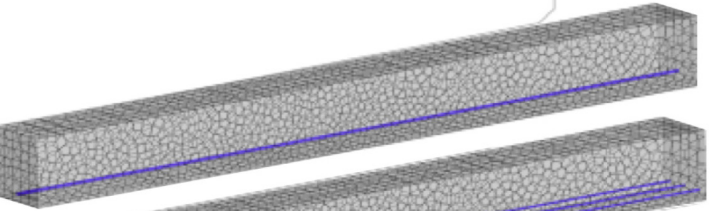

(c)

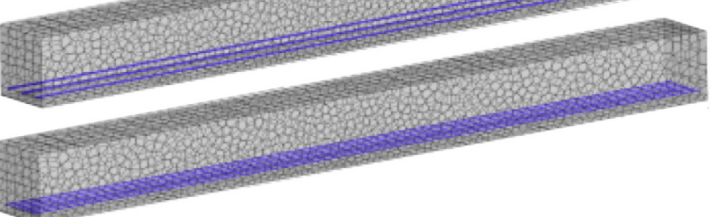

Figure 9. $\mathrm{RC}$ beam mesh configuration: (a) with a single rebar (case 1); (b) with three rebars (case 2); (c) with six rebars (case 3) [4]

(a)

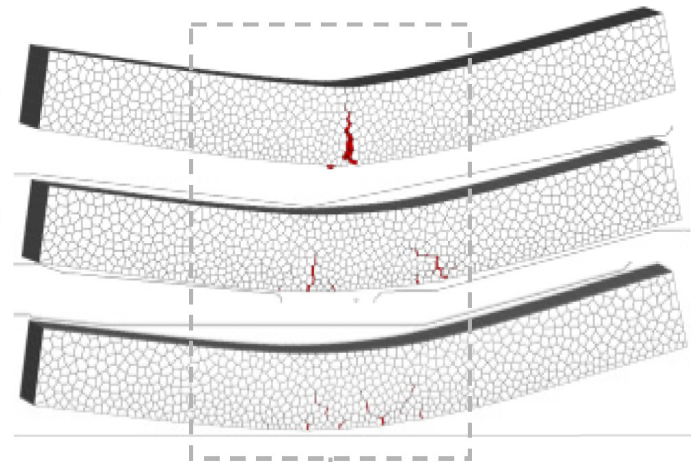

(a)

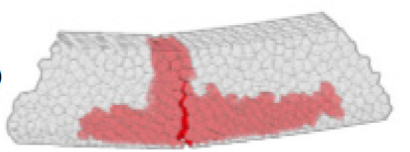

(b)

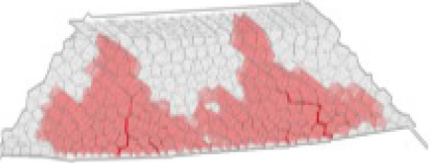

(c)

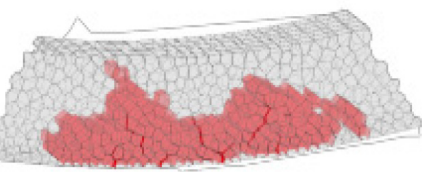

Figure 10. Failure configurations of RC beam during the simulations: (a) with single rebar; (b) with three rebars; (c) with six rebars [4]

\section{EXPECTATION OF EFFECTIVENESS}

The beta version of the "Virtual Concrete Structure Laboratory" was provided to the students to aid them in understanding the behavior of RC with different rebar distributions. In total, 28 (74\%) of the 38 students used the 
program. Fig. 11 shows the opinions of the students regarding the helpfulness of the program. Of the 28 students that used the program, 20 found it was helpful (72\%), 6 students found it somewhat helpful $(21 \%)$, and 2 students found it not helpful (7\%). Some responses from students who responded positively are listed below.

- What I learned in class didn't come to mind just by reading textbooks. It was interesting to use this program that shows the behavior of deformation. Just visualizing the beam was helpful.

- It would be more helpful if more content is developed.

- I could check if my answer is correct by comparing the results of hand calculations with the results from the program.

- It took considerable time to solve the problem by hand calculations, but using this program meant that the problem could be solved much faster.

In total, $93 \%$ of the students responded that the visualization part was helpful for enhancing their understanding. The survey shows that conducting a virtual experiment is as helpful as a real experiment for education purposes. The "Virtual Concrete Structure Laboratory," when completed, is expected to have a major role to play in improving the understanding of civil engineering by students.

\section{CONCLUSION}

This study described the development of a virtual laboratory platform to help the understanding of civil engineering course by students. Different types of concrete beam tests were conducted and the results were as expected. The linear structural analysis program allowed the students to understand linear elastic behavior by comparing the results of an analytical solution with those from numerical analysis. The concrete failure analysis program allowed the students to comprehend the behavior of concrete-like brittle material that shows the reason of reinforcement requirement in concrete. The RC analysis program explored the rebar distribution effect to show that if the rebars are distributed, the cracks are also distributed in a wide manner. Therefore, the students can understand the behavior of concrete and the reasons for the design code requirements for rebar distribution in an $\mathrm{RC}$ beam. Therefore, 'Virtual Concrete Structure Laboratory' is needed and will be helpful to students in understanding of concrete structure design requirements.

At present, only the beta version of 'Virtual Concrete Structure Laboratory' is available because the program is still being developed. In the near future, by the end of this project, we expect to provide positive results for the assessment of the 'Virtual Concrete Structure Laboratory' for engineering educational purposes.

\section{REFERENCES}

[1] B. Balamuralithara and P. C. Woods, "Virtual laboratories in English education: The simulation lab and remote lab," in Comput. Applicat. Eng. Educ., vol 17, pp.108-118, March 2009. http://dx.doi.org/10.1002/cae.20186

[2] H. P. Rossmanith, "Fracture and Damage of Concrete and Rock, FDCR-2," London: E\&FN Spon, November 1992.

[3] C. Zhang, P. Cao, Y. Cao and J. Li, "Using finite element software to simulation fracture behavior of three-point bending beam with initial crack," J. Software, vol. 8, pp.1145-1150, May 2013. http://dx.doi.org/10.4304/jsw.8.5.1145-1150

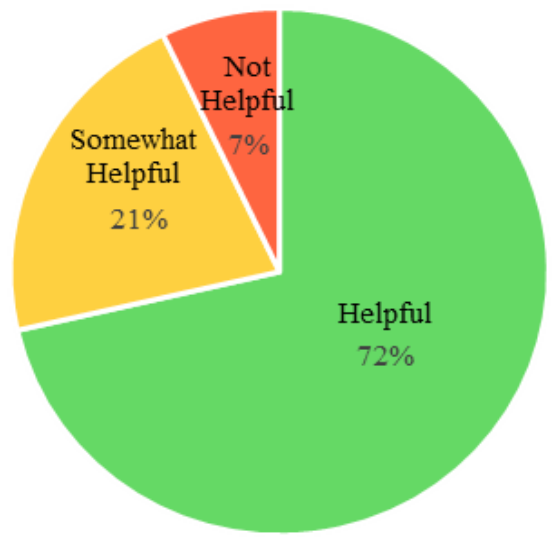

Figure 11. Student survey on helpfulness of the programs

[4] Y. K. Hwang, A. D. Adeba, J. E. Bolander, and Y. M. Lim. "Build-up virtual laboratory for reinforced concrete structures to enhance understanding design requirements," in Interactive Collaborative Learning (ICL), Florence, Italy, Sept 2015, pp. 330333.

[5] A. C. Bordelon, "Fracture behavior of concrete materials for rigid pavement systems," M.S. thesis, Dept. Civil Eng., Univ. of Illinois at Urbana-Champaign, Illinois, USA, 2007.

[6] J. E. Bolander and S. Saito, "Fracture anal. using spring networks with random geometry," Eng. Frac. Mech., vol. 61, pp.569-591, November-December 1998. http://dx.doi.org/10.1016/S00137944(98)00069-1

[7] M. Yip, J. Mohle, and J. E. Bolander, "Automated modeling of three-dimensional structural components using irregular lattices," Comput.-Aided Civ. Infrastruct. Eng., vol. 20, pp. 393-407, November 2005. $\quad$ http://dx.doi.org/10.1111/j.1467$\underline{\text { 8667.2005.00407.x }}$

[8] K. Kim, J. E. Bolander, and Y. M. Lim, "Failure simulation of RC structures under highly dynamic conditions using random lattice models," Comput. Struct., vol. 125, pp. 127-136, September 2013. http://dx.doi.org/10.1016/j.compstruc.2013.04.007

[9] K. Kim, "Development and application of nonlinear interface link elements within a three-dimensional random lattice model," M.S. thesis, Dept. Civil Eng., Yonsei Univ., Seoul, South Korea, December 2005.

[10] H. Lee, "Evaluation of structural performance and strengthening of corrosion deteriorated reinforced concrete members," ph.D. dissertation, Dept. Civil Eng., Tokyo Univ., Tokyo, Japan, 1997.

[11] J. M. Gere and B. J. Goodno, "Mechanics of Materials," Cengage Learning, 2012.

[12] J. E. Bolander and S. Berton, "Cohesive zone modeling of fracture in irregular lattices," Proceedings of Fracture Mechanics of Concrete, 2004.

\section{AUTHORS}

S. H. Park is with the Yonsei University, Seoul 120749 Korea (paulparksh@hanmail.net).

A. D. Adeba is with the Yonsei University, Seoul 120749 Korea (aberader@gmail.com).

Y. K. Hwang is with the Yonsei University, Seoul 120749 Korea (y_khwang@hanmail.net).

J. E. Bolander is with the University of California Davis, Davis, CA 95616 USA (jebolander@ucdavis.edu).

Y. M. Lim is with the Yonsei University, Seoul 120749 Korea (yunmook@yonsei.ac.kr).

This article is an extended and modified version of a paper presented at the International Conference on Interactive Collaborative Learning (ICL2015), held 20-24 December 2015, in Florence, Italy. This work is ongoing research supported by the EDISON (Education-research Integration through Simulation On the Net) Program through the National Research Foundation of Korea (NRF), funded by the Ministry of Science, ICT \& Future Planning (2014M3C1A6038855). Submitted, 10 February 2016. Published as resubmitted by the authors 28 February 2016. 